\title{
Distinct variants affecting differential splicing of TGFBR1 exon 5 cause either Loeys-Dietz syndrome or multiple self-healing squamous epithelioma
}

\author{
Takayuki Fujiwara $\cdot$ Norifumi Takeda ${ }^{1} \cdot$ Hironori Hara ${ }^{1} \cdot$ Hiroyuki Morita ${ }^{1} \cdot J^{\prime}$ Kishihara ${ }^{2} \cdot$ Ryo Inuzuka $^{3} \cdot$ \\ Hiroki Yagi ${ }^{1} \cdot$ Sonoko Maemura ${ }^{1} \cdot$ Haruhiro Toko ${ }^{1} \cdot$ Mutsuo Harada $^{1} \cdot$ Yuichi Ikeda ${ }^{1} \cdot$ Hidetoshi Kumagai $^{1}$. \\ Seitaro Nomura ${ }^{1} \cdot$ Eiki Takimoto $^{1} \cdot$ Hiroshi Akazawa $^{1} \cdot$ Junya Ako $^{2} \cdot$ Issei Komuro ${ }^{1}$
}

Received: 4 September 2017 / Revised: 12 January 2018 / Accepted: 13 February 2018 / Published online: 30 April 2018

(c) European Society of Human Genetics 2018

\begin{abstract}
Variants in TGFBRI have been reported to induce two completely distinct diseases, namely Loeys-Dietz syndrome (LDS) and multiple self-healing squamous epithelioma (MSSE). However, detailed mechanisms underlying this effect remain unknown. We report a Japanese familial case of LDS with a novel splice donor site variant in TGFBRI gene (c. $973+1 \mathrm{G}>$ A; NG_007461.1). The intronic variant was predicted to mediate in-frame exon 5 skipping within the serine/threonine kinase (STK) domain, which may also be mediated by a similar TGFBRI variant of a splice acceptor site in intron 4 (c.806-2 A > $\mathrm{C})$, identified in a British familial case of MSSE. Therefore, ex vivo splicing and functional assays were performed in mammalian cells to evaluate the effect of these sequence variants. The MSSE variant activated a cryptic acceptor site at 76 bp downstream of the $3^{\prime}$ natural splice acceptor site, which produced an out-of-frame transcript (r.807_882del, p. Asn270Thrfs*8). In contrast, the LDS variant generated two types of in-frame transcription products, r.[806_973del, 965_973 del], and produced two functionally inactivated proteins, p.[Asp269_Gln324del, Thr323_Gly325del], as a result of exon 5 skipping and the activation of a cryptic donor splice site at 9 bp upstream of the $5^{\prime}$ natural splice donor site, respectively. Our results support the previously proposed but not yet approved mechanism that dominant-negative and truncating variants in STK domain induce LDS and MSSE, respectively.
\end{abstract}

\section{Introduction}

Loeys-Dietz syndrome (LDS) is a heritable autosomal dominant disorder of the connective tissue, and patients with LDS present with several characteristics that are

These authors contributed equally: Takayuki Fujiwara, Norifumi Takeda.

$\triangle$ Norifumi Takeda

notakeda-tky@umin.ac.jp

1 Department of Cardiovascular Medicine, The University of Tokyo Hospital, 7-3-1 Hongo, Tokyo 113-8655, Japan

2 Department of Cardiovascular Medicine, Kitasato University School of Medicine, 1-15-1 Kitasato, Minami-ku, Sagamihara 252-0374, Japan

3 Department of Pediatrics, The University of Tokyo Hospital, 7-3-1 Hongo, Tokyo 113-8655, Japan similar to those of Marfan syndrome (MFS), including aortic aneurysm and dissection. However, patients with LDS are more likely to exhibit rapidly progressive aortopathy with a tendency for rupture and dissection in the aorta/arteries at a young age and at smaller dimensions [1$3]$. LDS is caused by a variant in genes encoding a component of the transforming growth factor- $\beta$ (TGF- $\beta$ ) signaling pathway, particularly TGFBR1 and TGFBR2, which encode transmembrane serine/threonine kinase (STK) receptors. In LDS, most variants in TGFBRI/2 genes are missense and are located in or immediately flanking the evolutionarily conserved STK domain $[4,5]$.

Recently, variants in TGFBR1 gene have been reported to induce a different type of disorder called multiple selfhealing squamous epithelioma (MSSE) [6]. MSSE is an autosomal dominant skin cancer syndrome characterized by the development of multiple rapidly growing invasive skin tumors that emerge for a few weeks only to subsequently spontaneously regress and heal with scarring. Such growths do not occur with LDS. Goudie et al. [6] have suggested 
molecular mechanisms by which variants in TGFBRI elicit two completely distinct diseases, namely LDS and MSSE. LDS is induced by dominant-negative missense variants within or near STK domain, whereas MSSE is induced by variants in the extracellular ligand-binding domain or truncating variants in STK domain.

In the present study, we report a Japanese familial case of LDS with a novel splice donor site variant in intron 5 $(c .973+1 \mathrm{G}>\mathrm{A})$. This variant is predicted to induce the inframe deletion of exon 5 within STK domain, exerting a dominant-negative effect, thus following the scenario suggested above. However, a similar variant that is expected to cause the in-frame deletion of exon 5 (c.806-2 A > C) has been reported to induce MSSE in a British familial case [6, 7]. In the present study, we describe a novel splicing mechanism by which these two apparently similar variants produce different system diseases.

\section{Materials and methods}

\section{Histological analysis}

Extended histological study of aortic aneurysmal tissues was approved by the University of Tokyo Hospital ethics committee (2233). The following antibodies were used: smooth muscle $\alpha$-actin (Sigma) and phospho-Smad2 (Cell Signaling Technology).

\section{Genetic analysis}

The genetic analysis of aortic aneurysms and dissections was approved by the University of Tokyo Hospital ethics committee (G-1538). Variant analyses of thoracic aortic aneurysm-related genes (FBN1, TGFBR1, TGFBR2, TGFB2, TGFB3, SMAD3, ACTA2, and MYH11) were performed using the Sanger sequencing method, as described previously [8]. The pathogenic variant of TGFBR1 (c.973 $+1 \mathrm{G}>\mathrm{A}$ ) and the patient's phenotype were submitted to the gene variant database www.LOVD.nl/TGFBR1 (Individual ID: 00144417).

\section{Splice analysis of TGFBR1 exon 5}

Total RNA was extracted from white blood cells, and cDNA synthesis was performed, as previously described [9]. Splicing patterns were analyzed using PCR with the following primers: forward, 5'-ggggagaagaagttgctgtt- $3^{\prime}$ and reverse, $5^{\prime}$-tgtcttactgccagtcctaagt- $3^{\prime}$, designed according to exons 4 and 6 sequences, respectively. The products were then subcloned into a pMD20T-vector using the Mighty TA-cloning Kit (TaKaRa) and sequenced for confirmation.

\section{Ex vivo splicing assay}

A genomic portion of TGFBR1 exon 5 of the proband (III-1) was amplified with $355 \mathrm{bp}$ of the $5^{\prime}$ and $261 \mathrm{bp}$ of the $3^{\prime}$ intronic flanking sequences using specific primers (forward, 5'-gggagatctaggaacagccactgagcacccaaccc-3' and reverse, 5'gggtctagaacctcagcccctgagtagctgggac $\left.-3^{\prime}\right)$ and cloned into the unique NdeI site of the $\alpha$-globin-fibronectin-extra domain B (EDB) splice reporter minigene (pTBNde(min), a gift from Franco Pagani) (Addgene \#15125). A construct with c.806-2 $\mathrm{A}>\mathrm{C}$ variant was generated through site-directed mutagenesis. The plasmids harboring these constructs were then transfected into the NIH3T3 cells using Lipofectamine 2000 (Thermo Fisher Scientific). Following RNA isolation and cDNA synthesis, RT-PCR analysis for the splicing pattern was performed as previously described [10], using the following primers: forward, 2-3 $\alpha 5^{\prime}$-caacttcaagctcctaagccactgc$3^{\prime}$ and reverse, B2 5'-ggtcaccaggaagttggttaaatca-3'.

\section{Generation of the TGFBR1 expression constructs}

The plasmid encoding a genetically manipulated human TGFBR1 mutant with intrinsic constitutive activity with a Cterminal HA-tag (pCMV5-TBR1-T204D-HA, abbreviated as construct CA) was purchased from Addgene (\#19162). The plasmid encoding human TGFBR1 with a C-terminal HA-tag (pCMV5-TBR1-HA, abbreviated as construct WT) was generated from construct CA by correcting the mutant amino acid to the wild-type through site-directed mutagenesis. Variant plasmids expressing a 9-bp deletion in exon 5 of TGFBR1 (c.965_973del) (pCMV5-TBR1ex5del9bp-HA and pCMV5-TBR1-T204D-ex5del9bp-HA, abbreviated as constructs Ex5del9 and CAEx5de19, respectively) were generated through site-directed mutagenesis. To generate exon 5 deletion variants (pCMV5-TBR1ex5del-HA and pCMV5-TBR1T204D-ex5del-HA, abbreviated as construct Ex5del and CAEx5del, respectively), cDNA fragments were PCRamplified using primers designed according to a sequence located upstream of the $X b a \mathrm{I}$ site in exon 4 (forward) and a complementary sequence containing the last coding sequences in exon 9 (reverse), with an HA-tag coding sequence and the BamHI site at the $3^{\prime}$ end. Then, the variant clones were

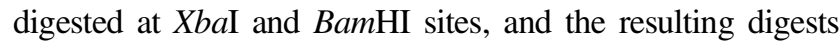
were subcloned into comparable vector sites of WT and CA, respectively. All TGFBR1 expression vectors expressed uniform high levels of proteins, as verified by Western blotting against TGFBR1 (Abcam) in HEK293T cells (Fig. 4a).

\section{Dual luciferase assays}

HEK293T cells were co-transfected with TGFBR1 expression vectors, SMAD3:SMAD4 inducible firefly luciferase reporter plasmid (pGL4.48 (luc2P/SBE/Hygro), Promega) and 


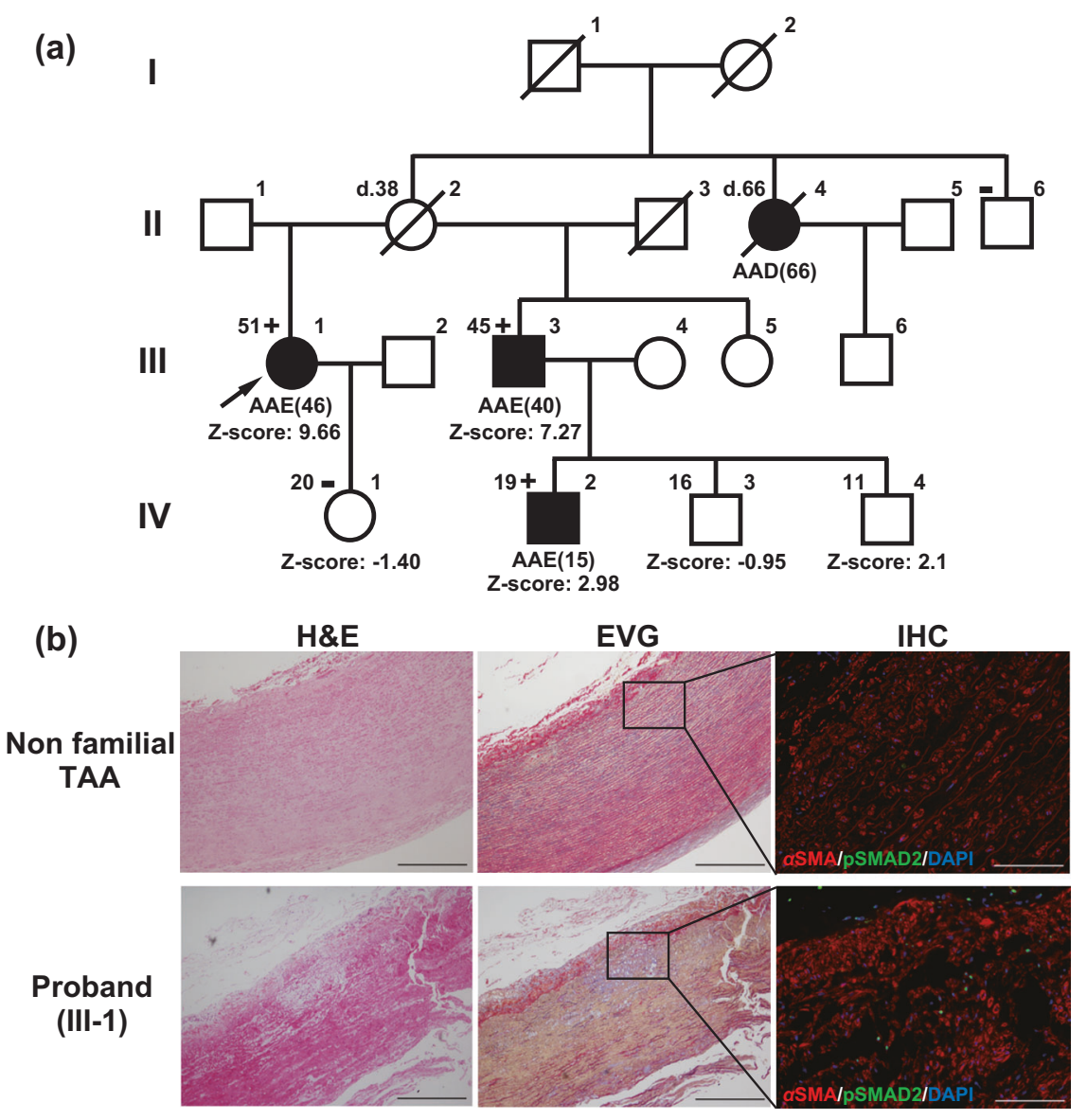

Fig. 1 Japanese familial case of LDS with aortic diseases. (a) Pedigree of the Japanese family case of LDS with clinical features. The age is shown in the upper left corner and "d" indicates the age at death. The numbers in parentheses are the age at diagnosis for aortic dissections or annuloaortic ectasia (AAE). The plus and minus symbols in the upper left corner indicate the presence and absence of the variant, respectively. AAD indicates acute aortic dissection. Symbols: circle, female; square, male; open symbol, unaffected; filled symbol, aortic aneurysm or dissection affected; diagonal line, died; $Z$-score, the aortic root $Z$-score. (b) Histological analysis of the surgically dissected aortic tissues of the proband and non-familial TAA patient aged 70 years. TGF- $\beta$ signaling was examined by immunohistochemical (IHC) staining against phosphorylated SMAD2. Scale bars: $500 \mu \mathrm{m}$ in H\&E and EVG; $100 \mu \mathrm{m}$ in IHC. DAPI 4', 6-diamidino-2-phenylindole for nuclear staining, $E V G$ Elastica van Gieson staining, $H \& E$ hematoxylin and eosin staining, $\alpha S M A$ alpha smooth muscle actin for smooth muscle cell staining, TAA thoracic aortic aneurysm constitutively active renilla luciferase plasmid (pRL-SV40, Promega) using Lipofectamine 2000. Luciferase fluorescence intensities were analyzed using the Dual-Glo Luciferase Assay System (Promega) and the GloMax-Multi Luminescence System (Promega). Reporter activities were normalized to the measured renilla luciferase activity of pRL-SV40.

\section{Result}

\section{Clinical report: familial LDS}

The proband (III-1) was a 51-year-old Japanese woman who had visited a university hospital for paroxysmal palpitations at the age of 46 years (Fig. 1a). During the evaluation, echocardiography revealed an enlargement of the sinuses of Valsalva (65 mm; Z-score [2], 9.66). The aneurysm was surgically treated through the David procedure. She was then referred to the Marfan clinic at the University of Tokyo Hospital because of a family history of aortic aneurysm and dissection; her aunt (II-4) had suddenly died from aortic dissection at the age of 66 years, and her younger half-brother (III-3) and his son (IV-2) presented with an aortic annuloectasia. Her height was $159 \mathrm{~cm}$, weight was $40 \mathrm{~kg}$, and she presented with a funnel chest, dolichodactyly, scoliosis, high-arched palate, skin striae, umbosacral dural ectasia, and tortuous cerebro-cephalic arteries. She suffered from the easy bruising of the skin and hypermenorrhea but had never developed skin tumors. Extended histological examinations revealed elastin degradation, cystic medial necrosis, and increased TGF- $\beta$ signaling in the aortic wall (Fig. 1b). 
(a)

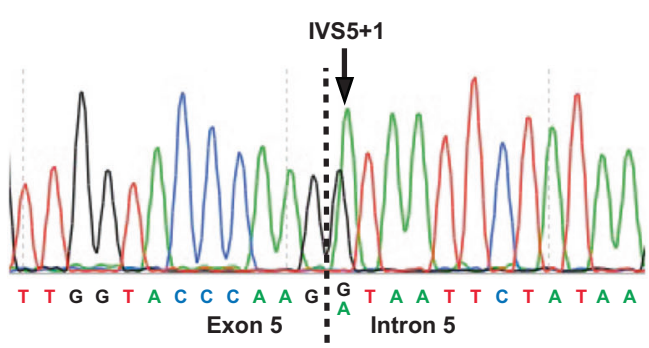

(b)
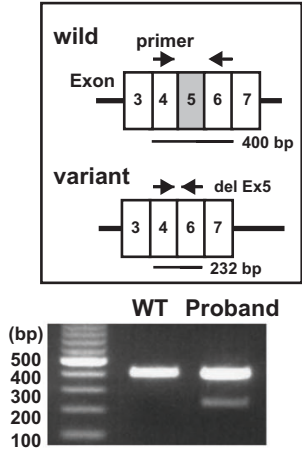

(c)

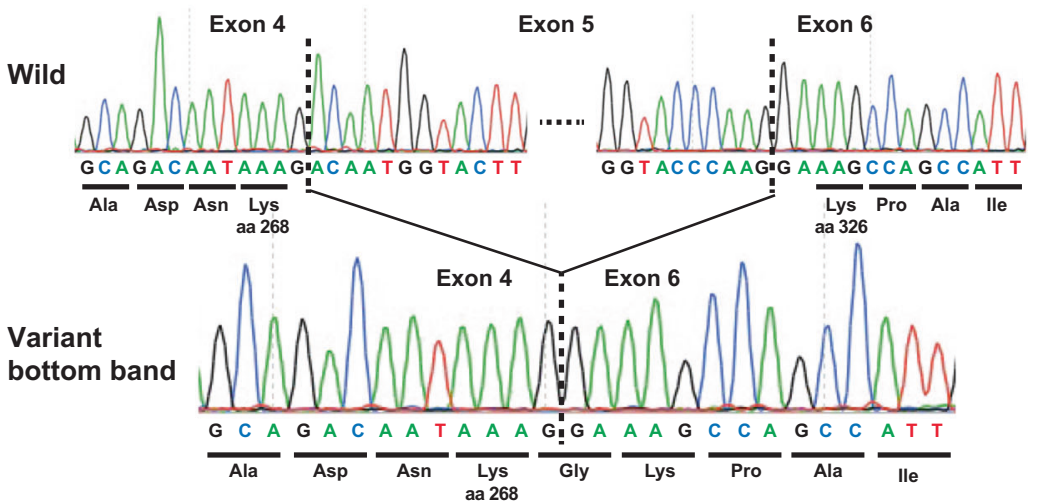

(d)

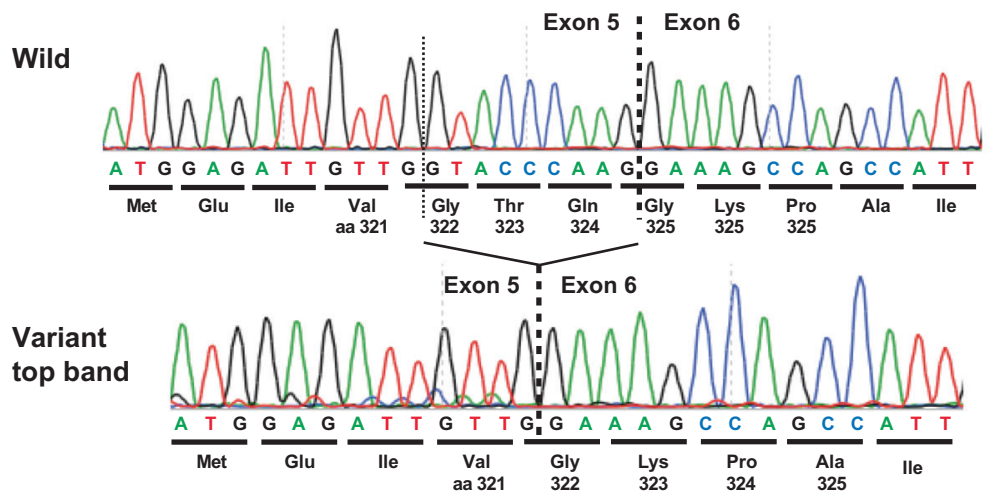

Fig. 2 TGFBR1 variant analysis. (a) Genomic DNA sequencing revealed a heterozygous single-base substitution (c. $973+1 \mathrm{G}>\mathrm{A}$ ) in the proband. (b-d) Splice analysis of exon 5. (b) Arrows indicate the position of PCR (top panel). Wild-type and variant cDNA were predicted to produce 400 bp and 232 bp fragments. The actual PCR products on 1.2\% agarose gel (bottom panel) in a healthy unaffected volunteer (left, WT) and the proband (right). The doublet bands in the gel indicate the splicing abnormality. (c, d) Direct sequencing of cDNA from wild-type (top) and variant (bottom) alleles in the proband revealed a 168 bp deletion corresponding to entire exon 5 skipping (c) and a last 9 bp deletion of exon 5 (d). aa amino acid position of TGFBR1

\section{Novel splice site variant (c.973 $+1 \mathrm{G}>$ A) of TGFBR1 gene}

We performed genetic analyses among her family members (affected: III-1, III-3, IV-2; unaffected: II-6, IV-1). We identified a novel splice donor site variant in intron 5 of TGFBRl gene $(\mathrm{c} .973+1 \mathrm{G}>\mathrm{A})$ in the three affected patients only (Fig. 2a), indicating that this cosegregated with a phenotype of aortic aneurysm and dissection.

\section{Effect of c.973 + $1 \mathrm{G}>$ A variant on mRNA splicing}

We next examined the transcriptional consequences using the cDNA of the proband generated from RNA of white blood cells. Given that c. $973+1 \mathrm{G}>$ A variant causes exon 5 skipping, PCR products of the wild-type and variants were predicted to be 400-bp and 232-bp fragments, respectively (Fig. 2b, top). Although no apparent extra bands were detected on electrophoresis (Fig. 2b, bottom), the direct sequencing of PCR products revealed two 
(a)

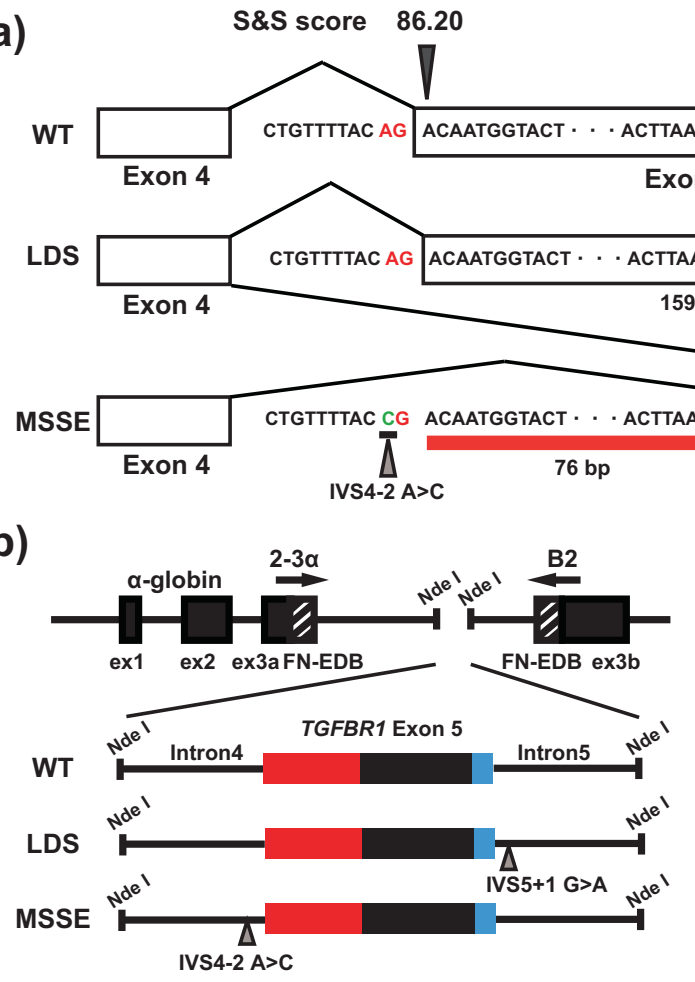

$\begin{array}{lll}83.12 & 58.41 & 87.85\end{array}$

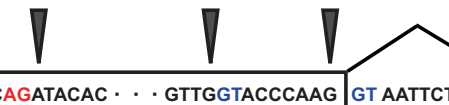
Exon 5 (168 bp)

Exon 6

(e)

Exon 6

(b)

(c)

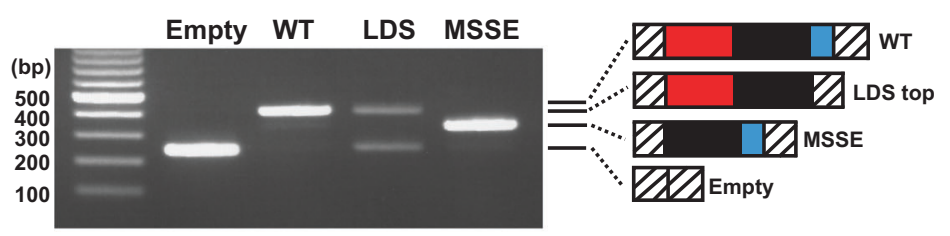

(d)

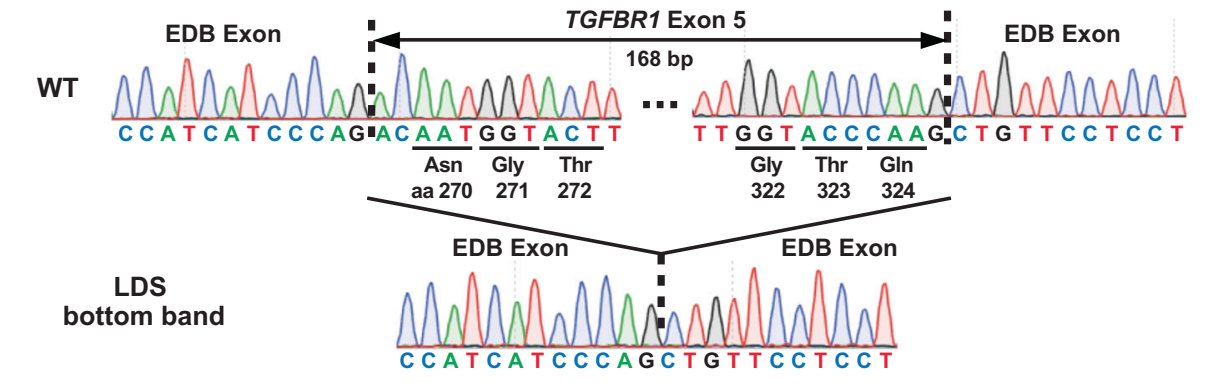

(f)

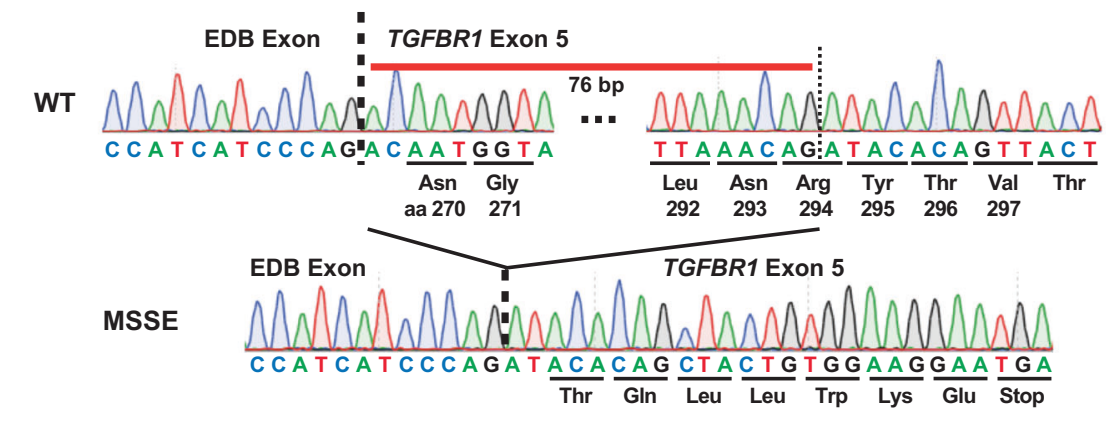


Fig. 3 The ex vivo splicing assay. (a) Natural and putative splice sites in the region around TGFBRI exon 5 with $\mathrm{S} \& \mathrm{~S}$ splice site scores. The putative splicing donor site $9 \mathrm{bp}$ upstream of the $5^{\prime}$ natural splice site and splice acceptor site $76 \mathrm{bp}$ downstream from the $3^{\prime}$ natural splice site were predicted to contribute to the pathogenesis of LDS and MSSE, respectively. (b) The pTBNde(min) minigene system used to analyze the effect of $T G F B R 1$ variants on ex vivo pre-mRNA splicing. (c) Splicing products amplified by RT-PCR using 2-3 $\alpha$ and B2 primers shown in (b) were separated by $1.2 \%$ agarose gel electrophoresis. The identity of the spliced products was established by direct sequencing (d-f) and is schematically represented on the right. (d-f) Direct sequencing of cDNA from wild-type (top) and variant (bottom) alleles. In the small and large products of LDS variant alleles, the entire exon 5 (168 bp) (d) and last 9 bases of exon 5 (e) were missing, respectively. (f) For the MSSE variant allele, the first $76 \mathrm{bp}$ bases of exon 5 was missing. FN-EDB, fibronectin-extra domain B; aa, amino acid position of TGFBR1

abnormal fragments of different sizes: a 232-bp fragment corresponding to entire exon 5 skipping (r.806_973del, p. Asp269_Gln324del; (Fig. 2c) and a 391-bp fragment generated by the loss of the last nine bases of exon 5 (r.965_973del, p.Thr323_Gly325del; Fig. 2d), which almost overlapped with the 400-bp wild-type products, as presented in Fig. 2b (bottom).

\section{Ex vivo splicing assay of two variants (c.973 $+1 \mathrm{G}>$ $A$ and C.806-2 A > C)}

These results encouraged us to verify the possibility of a difference in splicing patterns between patients with c.806-2 A > C (MSSE) or c. $973+1 \mathrm{G}>\mathrm{A}$ (LDS) variant. First, the strength of splice sites around exon 5 was calculated using the Shapiro-Senapathy (S\&S) splice scores (Fig. 3a) [11]. Two potential splice sites were identified within exon 5: one at 76 bp downstream of the $3^{\prime}$ natural splice acceptor site, with high (S\&S) splice scores (83.12), and another at $9 \mathrm{bp}$ upstream of the $5^{\prime}$ natural splice donor site, with low S\&S score (58.41). Next, functional ex vivo splicing assay was performed to evaluate the effect of sequence variants (Fig. 3b).

Analyses of the resulting splice products revealed that the wild-type and variant constructs produced differentially spliced products (Fig. 3c). The wild-type construct produced a transcript in which exon 5 was correctly spliced to EDB exons. In contrast, the c. $973+1 \mathrm{G}>\mathrm{A}$ variant produced two products resulting from splicing at approximately $400 \mathrm{bp}$ (almost the same as the wild-type) and $237 \mathrm{bp}$ (the vector exons alone, indicating exon 5 skipping), and the c.806-2 A > C variant produced a transcript resulting from splicing at approximately $350 \mathrm{bp}$. These alternatively spliced products were submitted to direct sequencing to establish their identity. The results demonstrated that the c. $973+1 \mathrm{G}>$ A variant mediated exon 5 skipping (Fig. 3d) and activated a cryptic donor splice site at $9 \mathrm{bp}$ upstream of the $5^{\prime}$ natural splice donor site (Fig. 3e), indicating that the c. $973+1 \mathrm{G}>\mathrm{A}$ variant allele generated two in-frame transcripts, r.[806_973del, 965_973del] encoding two proteins, p.[Asp269_Gln324del, Thr323_Gly325del], respectively. In contrast, the c.806- $2 \mathrm{~A}>\mathrm{C}$ variant activated a cryptic acceptor site at $76 \mathrm{bp}$ downstream of the $3^{\prime}$ natural splice acceptor site, producing an out-of-frame transcript (r.807_882del, p.Asn270Thrfs*8; Fig. 3f).

\section{Effect of $c .973+1 \mathrm{G}>$ A variant on in vitro TGF- $\beta$ / Smad signaling pathway}

Two in-frame deletion proteins partially lacking STK domain from c. $973+1 \mathrm{G}>\mathrm{A}$ variant allele may have the potential to alter TGF- $\beta$ signal transduction. Thus, a vector expressing wild-type or variant TGFBR1 was transfected into HEK293T cells with low endogenous TGFBR1 levels to examine effects c. $973+1 \mathrm{G}>\mathrm{A}$ variant on Smadbinding element (SBE) luciferase reporter (Fig. 4a).

After treatment with TGF- $\beta$, SBE luciferase activities in WT-transfected HEK293T cells were abolished in Ex5del and Ex5del9 transfected cells (Fig. 4b). In addition, the high constitutive activity of STK in CA-transfected HEK293T cells was completely inhibited in CAEx5del and partially inhibited in CAEx5del9 cells (Fig. 4c). These results indicate that two LDS-inducing proteins, p. [Asp269_Gln324del, Thr323_Gly325del], exert dominantnegative effects, at least in vitro.

\section{Discussion}

In this report, we described a familial case of LDS with a novel splice donor site variant of exon 5 in TGFBR1 gene (c.973 + $1 \mathrm{G}>\mathrm{A}$ ). To our knowledge, this is the first case of LDS with a splice site variant in the TGFBRl gene. The variant produced two types of in-frame but functionally inactivated proteins as a result of exon 5 skipping and the activation of a cryptic donor splice at a site 9 bp upstream of the $5^{\prime}$ natural splice donor site. Previously, a splice acceptor site variant of exon 5 (c.806-2 A > C) has been reported in a British familial case of the inherited skin cancer syndrome MSSE, without LDS-like phenotypes [6, 7]. This variant also has been predicted to result in exon 5 skipping. Our ex vivo splice analysis strongly suggested that c.806-2 A > $\mathrm{C}$ variant activated a cryptic acceptor site at $76 \mathrm{bp}$ downstream of the $3^{\prime}$ natural splice acceptor site, consequently producing an out-of-frame transcript (r.807_882del, p. Asn270Thrfs*8). Our results are consistent with previous observations that truncating variants in STK domain induce MSSE phenotypes and that missense variants within or near STK domain lead to LDS phenotypes [6].

Genetic variants in intronic splice sites account for approximately $10 \%-15 \%$ of human genetic diseases [12]. Most cases result in exon skipping because of a variant in 


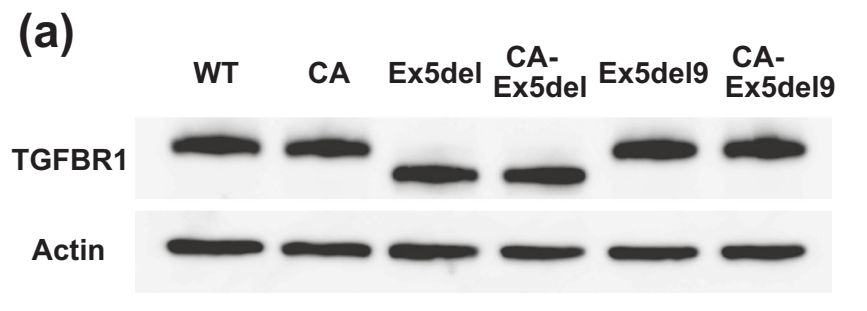

(b)
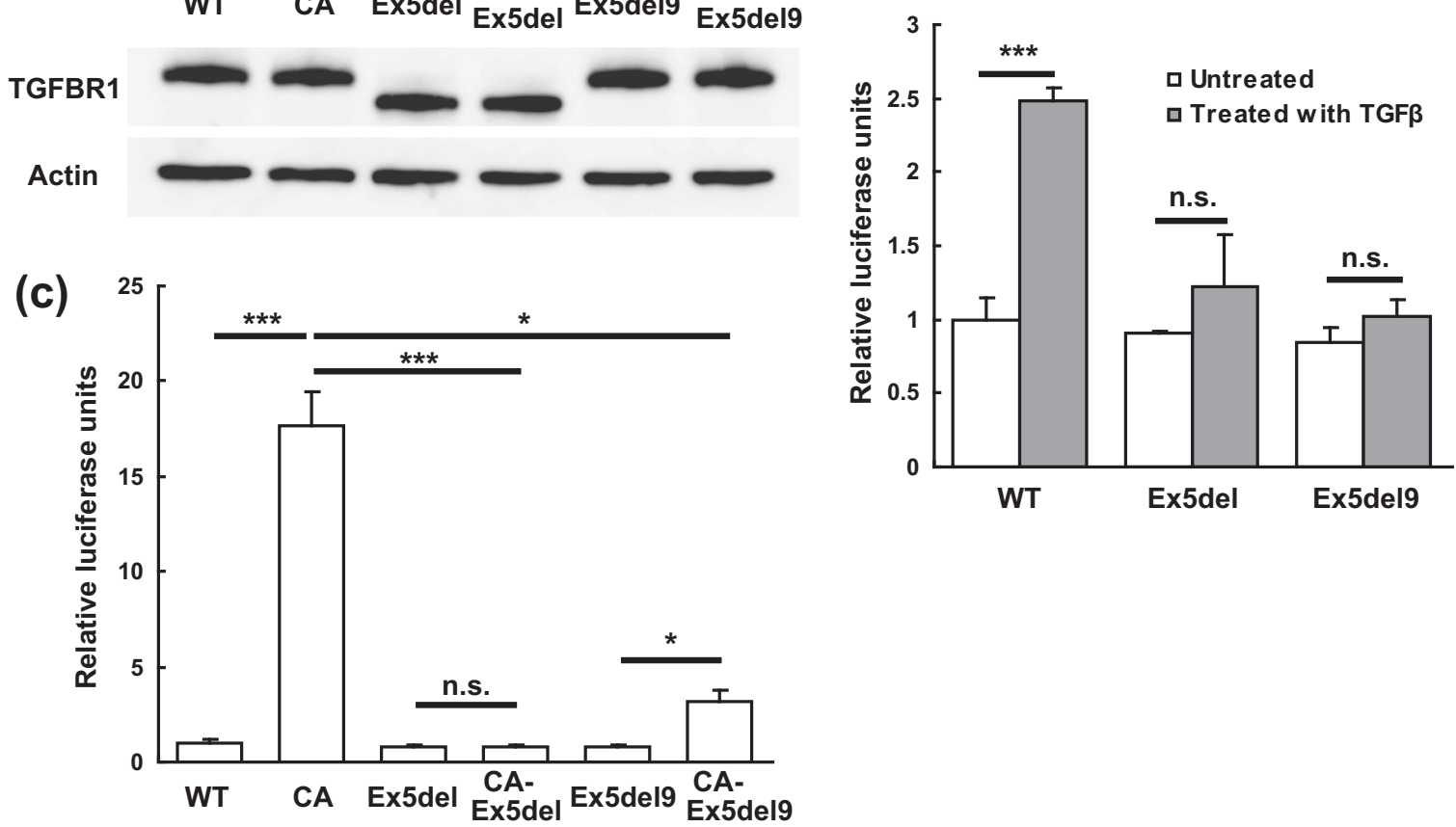

Fig. 4 Analysis of TGF- $\beta$ signaling by dual luciferase assay. (a) Western blot analysis for TGFBR1 expressions from generated constructs. (b, c) SBE-firefly luciferase activity in HEK293T cells. (b) HEK293T cells transfected with the indicated constructs were cultured for $36 \mathrm{~h}$, and after $4 \mathrm{~h}$ starvation with FCS-free DMEM, the cells were exposed to recombinant human TGF- $\beta 1$ (Wako) at a concentration of $5 \mathrm{ng} / \mathrm{mL}$ for $8 \mathrm{~h}$. $n=3$, *** $p<0.001$ versus the untreated-control (Student's $t$-test). (c) HEK293T cells were transfected with the indicated constructs and harvested for 48 $\mathrm{h}$ after transfection. $n=3, * * * p<0.001, * p<0.05$ (Analysis of Variance test)

the original splice site. However, some variants can activate a cryptic splice site, causing aberrant pre-mRNA splicing processes resulting in the subsequent generation of functionally modified proteins. However, to the best of our knowledge, two distinct system and/or organ diseases induced by the differential splicing of the same exon have not been reported to date. In contrast, variant types and locations within a single gene are known to affect the severity, age of onset, and rate of progression of the same organ diseases [13], and these are often referred to as different syndromes. Truncating and missense variants in the dystrophin gene $D M D$ induce severe Duchenne and mild Becker muscular dystrophy, respectively [14]. Further, variants in NFIX gene induce two different types of disorders affecting skeletal and neural developments; most NFIX variants induce Malan syndrome cluster in the evolutionarily conserved $N$-terminal DNA-binding/dimerization domain (exons 2 and 3), and some NFIX variants are associated with the Marshall-Smith syndrome cluster at the C-terminal end (exons 6-10) [15]. The detailed mechanism by which variants in TGFBRI induce two completely distinct diseases, namely LDS and MSSE, needs to be further investigated.

Most variants in the TGFBR1/2 genes inducing LDS have been verified in vitro and/or have been predicted to be loss-of-function variants [4, 5], whereas a paradoxical increase in TGF- $\beta$ activity has been observed in the aortic wall [16]. Although this paradox holds true in the present case (Figs. 1b, 4b-c), the underlying mechanism remains unknown. We and other researchers have speculated that TGF- $\beta$ signaling via the remaining wild-type receptors might play active roles in the development of TGF- $\beta$ vasculopathies because TGFBR1/2 functions as a homodimer receptor which maintains vascular homeostasis and activates many cellular processes [17].

In conclusion, we described a Japanese familial case of LDS with a novel splice donor site variant of the TGFBRI gene (c. $973+1 \mathrm{G}>\mathrm{A})$ and elucidated the unique mechanism by which distinct variants affecting the differential splicing of TGFBRI exon 5 induce either LDS or MSSE. Our results support the previously proposed but not yet validated mechanism that LDS and MSSE are induced by dominant-negative and truncating variants in STK domain, respectively, and also demonstrate the limits of in silico prediction tools for intronic variations. Therefore, further investigations are crucial to clarify the clinical and molecular significance of splice site variants.

Acknowledgements We would like to thank the patient and her family for their participation in this study. This study was supported by a 
Grant-in-Aid for Research on Rare and Intractable Diseases from the Japan Agency for Medical Research and Development (N.T.).

\section{Compliance with ethical standards}

Conflict of interest The authors declare no conflict of interest.

\section{References}

1. Loeys BL, Chen J, Neptune ER, et al. A syndrome of altered cardiovascular, craniofacial, neurocognitive and skeletal development caused by mutations in TGFBR1 or TGFBR2. Nat Genet. 2005;37:275-281.

2. Roman MJ, Devereux RB, Kramer-Fox R, O'Loughlin J. Twodimensional echocardiographic aortic root dimensions in normal children and adults. Am J Cardiol. 1989;64:507-512.

3. Loeys BL, Schwarze U, Holm T, et al. Aneurysm syndromes caused by mutations in the TGF-beta receptor. N Engl J Med. 2006;355:788-798.

4. Cardoso S, Robertson SP, Daniel PB. TGFBR1 mutations associated with Loeys-Dietz syndrome are inactivating. J Recept Signal Transduct Res. 2012;32:150-155.

5. Horbelt D, Guo G, Robinson PN, Knaus P. Quantitative analysis of TGFBR2 mutations in Marfan-syndrome-related disorders suggests a correlation between phenotypic severity and Smad signaling activity. J Cell Sci. 2010;123:4340-4350.

6. Goudie DR, D'Alessandro M, Merriman B, et al. Multiple selfhealing squamous epithelioma is caused by a disease-specific spectrum of mutations in TGFBR1. Nat Genet. 2011;43:365-369.

7. Robertson SJ, Bashir SJ, Pichert G, Robson A, Whittaker S. Severe exacerbation of multiple self-healing squamous epithelioma (Ferguson-Smith disease) with radiotherapy, which was successfully treated with acitretin. Clin Exp Dermatol. 2010;35: e100-e102.

8. Takeda N, Morita H, Fujita D, et al. A deleterious MYH11 mutation causing familial thoracic aortic dissection. Hum Genome Var. 2015;2:15028.

9. Hara H, Takeda N, Morita H, et al. Three novel BMPR2 mutations associated with advanced pulmonary arterial hypertension. Hum Genome Var. 2017;4:17010.

10. Muro AF, Caputi M, Pariyarath R, Pagani F, Buratti E, Baralle FE. Regulation of fibronectin EDA exon alternative splicing: possible role of RNA secondary structure for enhancer display. Mol Cell Biol. 1999;19:2657-2671.

11. Shapiro MB, Senapathy P. RNA splice junctions of different classes of eukaryotes: sequence statistics and functional implications in gene expression. Nucleic Acids Res. 1987;15: $7155-7174$.

12. Stenson PD, Mort M, Ball EV, et al. The Human Gene Mutation Database: 2008 update. Genome Med. 2009;1:13.

13. Zhu X, Need AC, Petrovski S, Goldstein DB. One gene, many neuropsychiatric disorders: lessons from Mendelian diseases. Nat Neurosci. 2014;17:773-781.

14. Takeshima Y, Yagi M, Okizuka Y, et al. Mutation spectrum of the dystrophin gene in 442 Duchenne/Becker muscular dystrophy cases from one Japanese referral center. J Hum Genet. 2010;55:379-388.

15. Malan V, Rajan D, Thomas S, et al. Distinct effects of allelic NFIX mutations on nonsense-mediated mRNA decay engender either a Sotos-like or a Marshall-Smith syndrome. Am J Hum Genet. 2010;87:189-198.

16. Akhurst RJ. The paradoxical TGF-beta vasculopathies. Nat Genet. 2012;44:838-839.

17. Takeda N, Yagi H, Hara H, et al. Pathophysiology and Management of Cardiovascular Manifestations in Marfan and Loeys-Dietz Syndromes. Int Heart J. 2016;57:271-277. 